\title{
A Double Iteration Greedy Heuristic Approach for Permanent Brachytherapy Planning
}

\author{
Bin Liang1 ${ }^{*}$, Fugen Zhou ${ }^{1}$, Bo Liu ${ }^{1}$, Jianguo Zhang ${ }^{2}$, Junjie Wang ${ }^{3}$, Yong $\mathrm{Xu}^{4}$ \\ 1 Image Processing Center, Beihang University, Beijing, China. \\ 2 Department of Oral and Maxillofacial Surgery, Peking University School and Hospital of Stomatology, \\ Beijing, China. \\ ${ }^{3}$ The Center of Oncology, Peking University Third Hospital, Beijing, China. \\ ${ }^{4}$ Department of Urology, the General Hospital of PLA, Beijing, China \\ * Corresponding author. Tel.: +861082338048; email: leangbin@gmail.com \\ Manuscript submitted September 2, 2014; accepted December 23, 2014. \\ doi: 10.17706/ijapm.2015.5.1.48-59
}

\begin{abstract}
This paper presents a double iteration greedy heuristic (DIGH) approach for permanent brachytherapy treatment planning. The DIGH approach adopts a greedy heuristic seed selection (GHSS) procedure to obtain a preliminary plan. In this process, the potential seeds are evaluated according to their ability to irradiate target volume while sparing organs-at-risk (OARs). Their impact on dosimetric homogeneity within target volume is also taken into account. The preliminary treatment plan generated by the GHSS procedure is further refined by the double iteration (DI) procedure. The DI procedure removes the needles containing only one seed (single seed) and implements the GHSS procedure again to obtain a temporary plan. The DI procedure terminates when the needle number of the temporary plan does not decrease. This process is guided by constantly removing the undesired part rather than imposing extra constrains. The efficiency and robustness of the DIGH approach is tested on three kinds of typical patient cases. For these cases, treatment plans are generated in less than 30s. The dosimetric distribution of these treatment plans achieves satisfactory dosimetric distribution. The numbers of used needles are kept within acceptable level. The experimental results demonstrate that the DIGH approach is fast, effective and robust. It has the potential to be used for intraoperative brachytherapy treatment planning in operation room.
\end{abstract}

Key words: Greedy heuristic, permanent brachytherapy, treatment planning.

\section{Introduction}

Permanent brachytherapy has been proven an effective approach for the tumors located in prostate [1]-[4], thoracic cavity [5]-[8], head and neck [9]-[11] etc. In the operation, radioactive seeds are directly implanted into target volume via template and puncture needles. The energy emitted by commonly used seeds attenuates rapidly along with the distance. The dosimetric distribution within region of interest (ROI) greatly depends on the positions of implanted seeds. Hence treatment plans, which determine the positions of implanted seeds, directly affects operation outcome. Generally, the goals of treatment planning include: 1 . delivering sufficient and homogenous dose to target volume; 2 . keeping the dose delivered to organs-at-risk (OARs) below harmful level.

The conventionally manual planning is a trial and error process: feasible plans are obtained by iteratively adjusting the positions of radioactive seeds, which is rather labor-intensive and time-consuming. To address this issue, optimization approaches are introduced to treatment planning. Currently available optimization 
methods are mainly developed for prostate brachytherapy treatment planning. These methods can be classified into stochastic, deterministic and heuristic approaches.

Stochastic approaches are proposed by J. Pouliot [12], Y. Yu [13], [14] and G. Yang [15] et al. These approaches are based on either simulated annealing (SA) or genetic algorithm (GA). Deterministic approaches were reported by E. Lee [16], [17] and D'souza et al. [18]. Treatment planning is modeled as a mixed integer-programming (MIP) problem. And the problem is solved by branch-and-bound (BB) approach. Heuristic approaches were reported by S. Yoo [19], [20], V. Chaswal et al. [21], [22]. For each step, the potential seeds are evaluated according to its ability to irradiate target volume while spare OARs, and the optimal seed is selected until sufficient dose is delivered to target volume. An isodose surface based constraint is used to prevent selected seed from congregating. In order to limit the number of used needles, S. Yoo confines the search space within existing needles when needle number reaches the predetermined threshold. V. Chaswal defines a penalty function for the seed requiring adding a new needle.

In our previous work [23], we developed an improved seed evaluation criterion and a flexible strategy to limit the number of puncture needles on the base of S. Yoo's work. In this paper, we adopted a more effective double iteration strategy (DI) to reduce the puncture needles. As this method is more generalized, it is suitable for prostate brachytherapy treatment planning, as well as adenoid and mediastinum planning.

\section{Method and Materials}

The double iteration greedy heuristic (DIGH) approach uses a greedy heuristic seed selection (GHSS) procedure to obtain a temporary plan (plan $\mathrm{T}$ ). Then plan $\mathrm{T}$ is refined by the double iteration (DI) procedure. The flowchart is shown in Fig. 1.

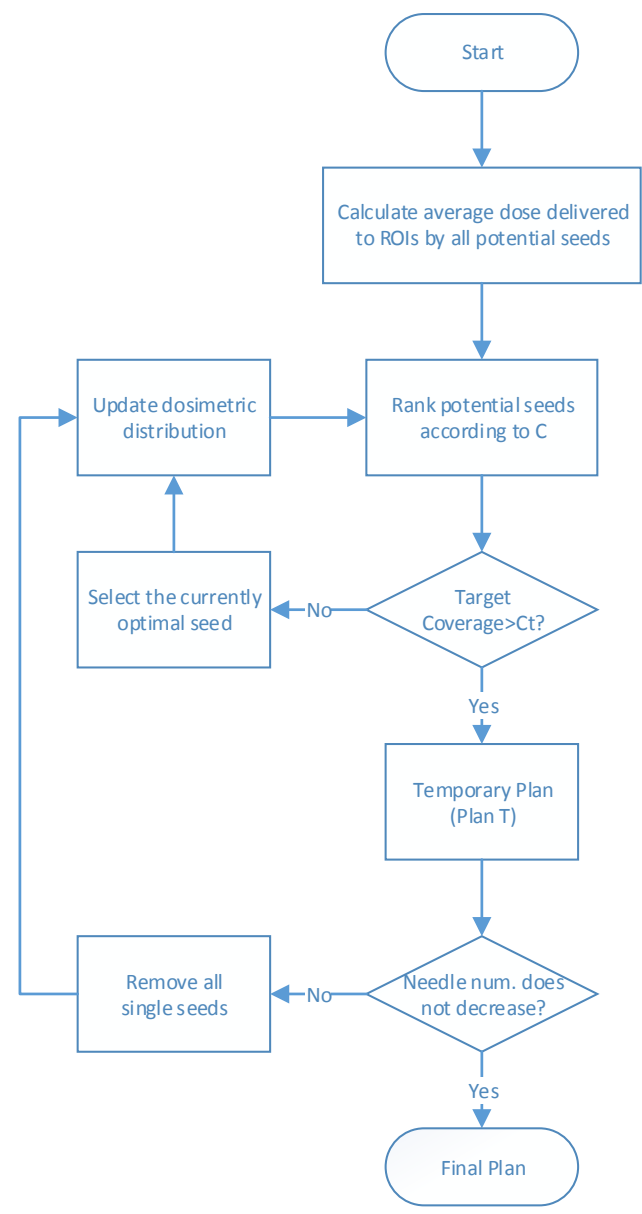

Fig. 1. The flowchart of the DIGH approach. 
The DIGH approach starts with calculating the average dose delivered to region of interest (ROI) by all the potential seeds. Then the GHSS procedure is implemented. For each step, the potential seeds are assessed by the evaluation criterion (C). The seed with the minimum value is considered as the currently optimal seed. The GHSS procedure terminates based on the current target coverage, which is defined as the percentage of target volume covered by the prescription dose (Dp). The currently optimal seeds are selected until the target coverage is equal to or greater than the predetermined threshold $(\mathrm{Ct})$. When the GHSS procedure terminates, the selected seeds construct plan T, which is further refined by the DI procedure. The DI procedure starts with removing the puncture needles carrying only one seed (single seed) of plan T. Based on the updated dosimetric distribution, a temporary plan is generated after the GHSS procedure is implemented again. Then the needle number of newly obtained plan is compared with previous plan. The DI procedure repeats until the number of puncture needle does not decrease.

\subsection{Seed Evaluation Criterion}

It has been demonstrated that the mean dose delivered to ROIs by one potential seed reflects its ability to irradiate these organs [19]. As the objective is to deliver sufficient and uniform dose to target volume while spare OARs, $C$ is defined as:

$$
C=\frac{\sum w_{i} \times \overline{O A R_{i}}}{\bar{T}} \times D
$$

where $\overline{O A R}$ and $\bar{T}$ are the mean dose value delivered to OARs and target volume by one seed. $w_{i}$ is the weighting factor for each OAR, which can be adjusted according to its sensitivity. D is dose deviation within target volume after the seed is implanted.

The mean dose ratio takes advantage of the prior knowledge about the relative position of potential seed and ROIs. Ranking according to this ratio, potential seeds, which are close to the center of target while far away from OARs, have higher priority to be selected. The weighting factor D excludes possible seeds near selected ones, which acts as a resistant force to prevent seed agglomeration.

\subsection{Double Iteration}

The GHSS procedure does not restrict the number of puncture needles. It is very possible that plan $\mathrm{T}$ uses more puncture needles than feasible level. The most unfeasible part of plan $\mathrm{T}$ is that the needles carrying only one seed (single seed). The DI strategy aims to replace these single seeds with the ones in the existing needles. The DI procedure starts with removing all single seeds of plan $\mathrm{T}$. Then the dosimetric distribution is updated by subtracting the corresponding dose value. Based on the updated dosimetric distribution, the GHSS procedure is implemented again to generate a temporary plan. The DI procedure repeats until the number of puncture needles does not decrease.

As the GHSS procedure always selects the optimal seed based on current dosimetric distribution, the selected seeds are not necessarily optimal for different dosimetric distribution. The removed single seeds at the beginning stage may not be optimal for the updated dosimetric distribution. On the other hand, the seeds in existing needles are kept and remain valid once selected. The DI process is guided by continuously removing the undesired part from the intermediate solutions rather than imposing extra constraints. Thus puncture needles are reduced without introducing undesired change to dosimetric distribution.

\section{Experiment and Results}

We tested the DIGH approach on the data of patients who had prostate, adenoid or mediastinum cancer. The currently available methods are mainly proposed for prostate brachytherapy planning. Among 
currently available methods, the heuristic $(\mathrm{YH})$ method presented in [20] is able to generate a feasible treatment plan using shorter time, and the deterministic (BB) method presented in [18] has the ability to guarantee the quality of treatment plan. So we implemented the two methods using the same patient data for comparison. Note that: we limit the computational time of the BB approach to two hours; otherwise it may take rather long time to complete.

For the case of prostate cancer, the planning image is transrectal ultrasound (TRUS) image with 5mm interval. For adenoid and mediastinum cancer, the planning image is CT image with $2.5 \mathrm{~mm}$ and $5 \mathrm{~mm}$ interval, see Table 1. For prostate brachytherapy, the template is fixed in the anterior-posterior (AP) direction. For adenoid and mediastinum, the direction is lateral, which can to be adjusted to avoid or minimize bone blocking. The sampled voxel spacing (SVS) are also listed in Table 1. For termination condition, the target coverage is all set to $98 \%$ for the three cases.

Table 1. Critical Parameters of the Patient Cases

\begin{tabular}{|c|c|c|c|c|c|c|c|c|}
\hline \multirow{2}{*}{ Patient case } & \multicolumn{2}{|c|}{ Planning image } & \multicolumn{2}{|c|}{ Template } & \multirow{2}{*}{$\begin{array}{l}\mathrm{SVS} / \\
\mathrm{mm}^{3}\end{array}$} & \multirow{2}{*}{ OAR } & \multirow{2}{*}{$\begin{array}{l}\text { SA/ } \\
\text { mGy }\end{array}$} & \multirow{2}{*}{$\begin{array}{c}\mathrm{D}_{\mathrm{p}} / \\
\mathrm{Gy}\end{array}$} \\
\hline & Mod. & Interval & Direction & Adjustable & & & & \\
\hline Prostate & TRUS & $5 \mathrm{~mm}$ & $\mathrm{AP}$ & No & $1 \times 1 \times 5$ & Yes & 0.4 & 145 \\
\hline Adenoid & CT & $2.5 \mathrm{~mm}$ & Lateral & Yes & $2 \times 2 \times 2.5$ & No & 0.7 & 140 \\
\hline Mediastinum & CT & $5 \mathrm{~mm}$ & Lateral & Yes & $5 \times 5 \times 5$ & No & 0.7 & 110 \\
\hline
\end{tabular}

As the energy emitted by radioactive seeds attenuates rapidly along with distance, the dose delivered to surrounding OARs is marginal, especially to those organs which are not contiguous with target, as the case for adenoid and mediastinum. However, for the case of prostate brachytherapy, urethra and rectum locate in the center of and closely adjacent to prostate. The dose delivered to these OARs is directly related with morbidity. Therefore, OARs are taken into account only for prostate treatment planning. The weighting factors of urethra and rectum in evaluation criterion are set to 1 .

We used 125I for all the patients. The seed activity (SA) and Dp are listed in Table 1. Dose value is computed according to the principle proposed by the Interstitial Collaborative Working Group (ICWG 1990) and the American association of physicists in medicine (AAPM Task Group 43) [24], [25].

We adopt a series of critical parameters to assess the dosimetric distribution of treatment plans. For prostate treatment plan, these parameters are selected according to the criteria recommended by AAPM [3] For adenoid and mediastinum treatment plans, evaluation parameters include D100, D90, V100 and DNR. Besides, the dose volume histogram (DVH), seed and dosimetric distribution on each slice are also shown for evaluation.

\subsection{Prostate Treatment Plan}

The primary criteria for treatment planning recommended by AAPM [3] are listed in Table 2. D90, D10 and D2cc are defined as the minimum dose in the "hottest" certain percentage (90\% and 10\%) or certain size (2cc) of the volume. For target volume, if P100 is greater than 95\%, D90 will be surely greater than Dp [3]. The two criteria are equivalent to each other.

Table 2. The Recommended Criteria for Prostate Brachytherapy Planning

\begin{tabular}{c|c|c|c}
\hline Organ & Prostate & Urethra & Rectum \\
\hline Criterion & $\mathrm{D} 90 \geq \mathrm{Dp}=145 \mathrm{~Gy}(\mathrm{P} 100 \geq 95 \%)$ & $\mathrm{D} 10<150 \% \mathrm{Dp}=217.5 \mathrm{~Gy}$ & $\mathrm{D} 2 \mathrm{cc}<\mathrm{Dp}=145 \mathrm{~Gy}$ \\
\hline
\end{tabular}

To evaluate the dosimetric distribution more comprehensively, we also calculated the following critical parameters:

- DNR: dose non-uniformity ratio, defined as the ratio of target volume covered by $150 \%$ (V150) to $100 \%$ (V100) Dp [26]; 
- The percentage of urethra volume covered by $120 \% \mathrm{Dp}$ (V120);

- The percentage of rectum volume covered by $80 \% \mathrm{Dp}$ (V80).

These critical parameters along with needle and seed numbers, computational time are all listed in Table 3. Fig. 2 shows the DVHs of prostate, urethra and rectum. Fig. 3 demonstrates the dosimetric distribution and seed configuration on each slice. The unit of D90, D10 and D2cc is Gy, and the unit of V100, V120 and V80 is \%. The unit of time is second. The unit of volume is cc. Without specially denoted, the parameters appeared in the following tables use the same unit.

Table 3. Critical Parameters of Prostate Treatment Plan

\begin{tabular}{|c|c|c|c|c|c|c|c|c|c|c|c|c|}
\hline \multirow{2}{*}{ Patient } & \multirow{2}{*}{$\begin{array}{c}\text { Target } \\
\text { Vol. }\end{array}$} & \multicolumn{4}{|c|}{ Configuration } & \multicolumn{3}{|c|}{ Prostate } & \multicolumn{2}{|c|}{ Urethra } & \multicolumn{2}{|c|}{ Rectum } \\
\hline & & Method & Needle & Seed & Time & V100 & D90 & DNR & D10 & V120 & D2cc & V80 \\
\hline & \multirow{3}{*}{31.8} & $\mathrm{YH}$ & 21 & 54 & 59.2 & 99.3 & 169.7 & 0.487 & 169.7 & 4.0 & 100.1 & 23.8 \\
\hline & & $\mathrm{BB}$ & 18 & 54 & 7200.0 & 98.0 & 165.3 & 0.492 & 171.1 & 3.3 & 88.5 & 2.5 \\
\hline & & DIGH & 20 & 50 & 12.2 & 98.6 & 168.2 & 0.328 & 171.1 & 2.8 & 92.8 & 16.9 \\
\hline \multirow{3}{*}{2} & \multirow{3}{*}{47.1} & YH & 27 & 71 & 147.8 & 98.7 & 171.1 & 0.516 & 175.5 & 17.6 & 76.9 & 0.1 \\
\hline & & $\mathrm{BB}$ & 18 & 72 & 7200.0 & 99.2 & 165.3 & 0.487 & 166.8 & 0.5 & 82.7 & 0.5 \\
\hline & & DIGH & 23 & 64 & 46.5 & 96.4 & 162.4 & 0.337 & 172.6 & 8.9 & 68.2 & 0.0 \\
\hline \multirow{3}{*}{3} & \multirow{3}{*}{35.3} & YH & 24 & 58 & 122.6 & 99.1 & 168.2 & 0.522 & 214.6 & 28.3 & 98.6 & 27.9 \\
\hline & & $\mathrm{BB}$ & 36 & 57 & 7200.0 & 98.6 & 159.5 & 0.390 & 168.2 & 1.9 & 85.6 & 4.0 \\
\hline & & DIGH & 23 & 51 & 16.9 & 97.3 & 161.0 & 0.316 & 172.6 & 5.6 & 100.1 & 35.1 \\
\hline \multirow{3}{*}{4} & \multirow{3}{*}{32.0} & YH & 17 & 54 & 6.0 & 99.5 & 171.1 & 0.499 & 178.4 & 18.3 & 78.3 & 1.6 \\
\hline & & BB & 15 & 56 & 7200.0 & 99.7 & 172.6 & 0.512 & 171.1 & 4.6 & 79.8 & 0.6 \\
\hline & & DIGH & 19 & 50 & 10.9 & 97.0 & 161.0 & 0.343 & 171.1 & 7.3 & 76.9 & 1.0 \\
\hline \multirow{3}{*}{5} & \multirow{3}{*}{32.5} & YH & 21 & 54 & 59.2 & 98.3 & 167.0 & 0.496 & 169.7 & 14.0 & 110.1 & 26.8 \\
\hline & & BB & 18 & 54 & 7200.0 & 98.2 & 163.4 & 0.461 & 173.2 & 6.8 & 84.6 & 4.5 \\
\hline & & DIGH & 19 & 50 & 12.2 & 98.5 & 164.5 & 0.370 & 174.5 & 5.6 & 98.2 & 18.0 \\
\hline
\end{tabular}

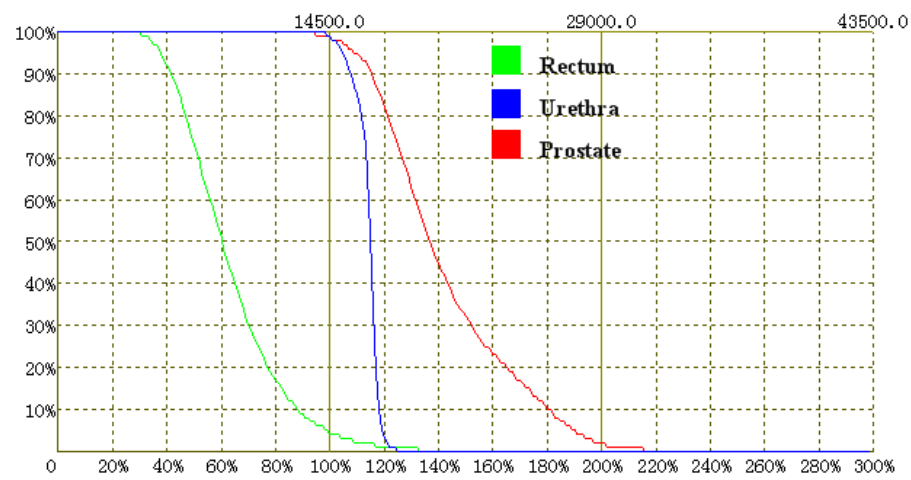

Fig. 2. The DVH of the treatment plan generated by the DIGH approach for patient 1 . Rectum urethra and prostate DVHs are depicted in green, blue and red respectively.

Compared with the $\mathrm{YH}$ approach, the DIGH approach yields better treatment plan in a shorter time. Compared with the BB approach, the DIGH plans achieve much better homogeneity. The BB approach protects OARs more effectively. However, the most obvious obstacle of using the BB approach for treatment planning the computational time. In [18], the approach is implemented using the 2D data. The computational time is 20-45 minutes, and it would be much longer if $3 \mathrm{D}$ data is used. In [20], the computational time is limited to 2 hours. The BB approach fails to generate treatment plans for two out of ten patent. Although, the hardware and the algorithm have been greatly improved since then, we assert it is very difficult to get a feasible treatment plan using the BB approach within several minutes. 

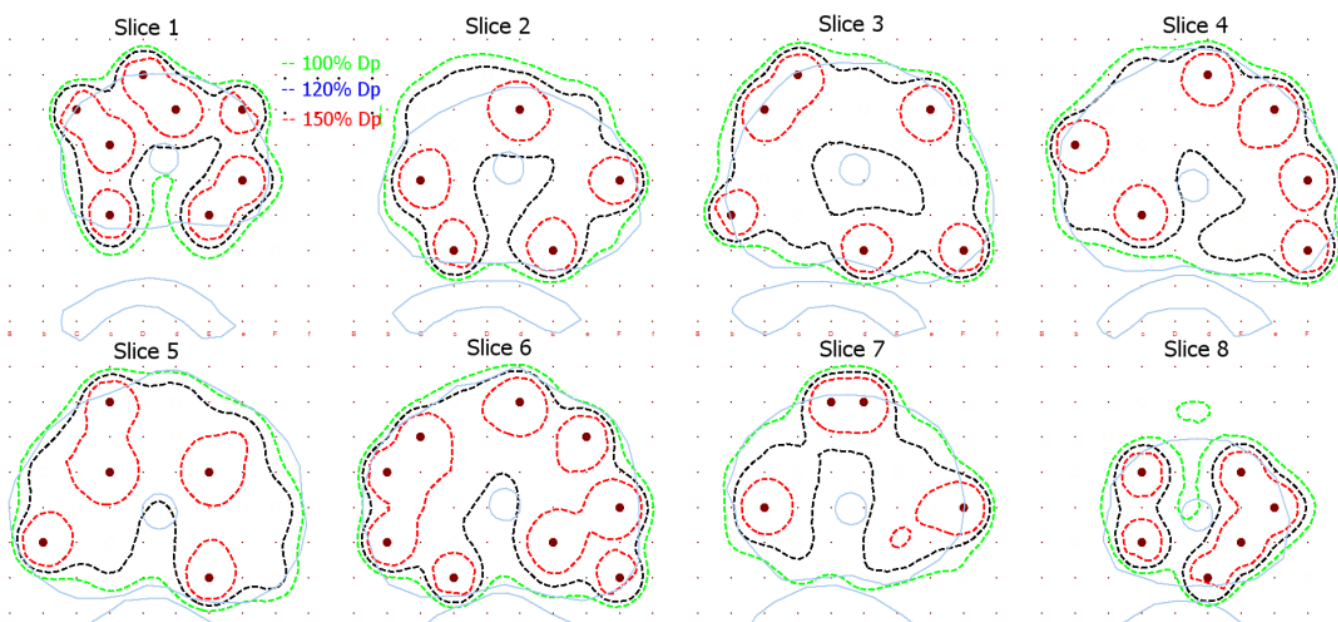

Fig. 3. Seed and dosimetric distribution of the treatment plan generated by the DIGH approach for patient 1. Target and OARs are depicted in solid lines on white background. The isodose lines of $100 \%, 120 \%$ and $150 \%$ Dp are depicted in green, blue and red, respectively. The selected seeds are marked by solid dots.

\subsection{Adenoid Treatment Plan}

For adenoid brachytherapy, the problem of bone blocking has to be taken into account. The direction of template needs to be adjusted, and the unfeasible seeds blocked by bones needs to be eliminated. Fig. 4 demonstrates the target contour, which is outlined by magenta solid line on one slice, potential seeds in yellow and the unfeasible seeds in red. The DIGH approach starts after the direction of template is determined and the unfeasible seeds are removed.

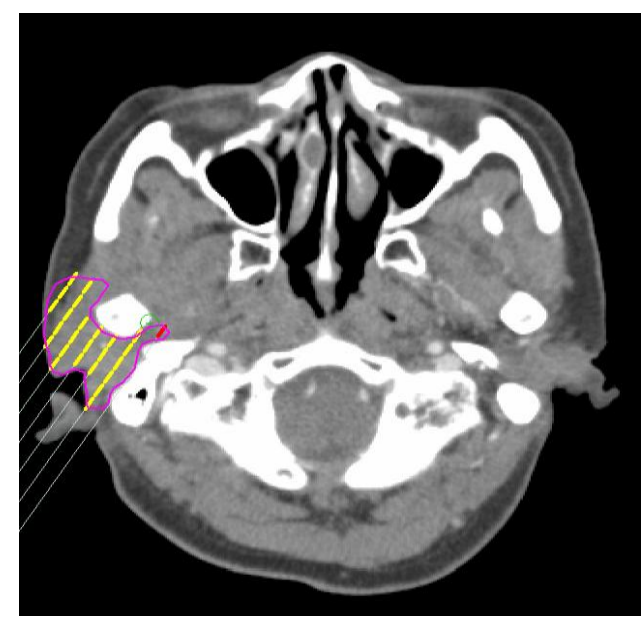

Fig. 4. Target contour, potential and unfeasible seeds.

Table 4. Critical Parameters of Adenoid Treatment Plans

\begin{tabular}{c|c|c|c|c|c|c|c|c}
\hline Patient & Vol. & V120 & V100 & DNR & D90 & Seed & Needle & Time \\
\hline 1 & 76.2 & 75.1 & 98.6 & 0.049 & 124.3 & 89 & 52 & 9.8 \\
\hline 2 & 64.6 & 65.1 & 99.3 & 0.038 & 130.2 & 72 & 43 & 8.4 \\
\hline 3 & 56.1 & 72.3 & 98.1 & 0.047 & 128.6 & 69 & 41 & 7.5 \\
\hline 4 & 78.5 & 61.4 & 99.2 & 0.052 & 132.3 & 90 & 54 & 10.3 \\
\hline 5 & 53.2 & 74.2 & 98.9 & 0.053 & 121.9 & 64 & 39 & 7.5 \\
\hline
\end{tabular}




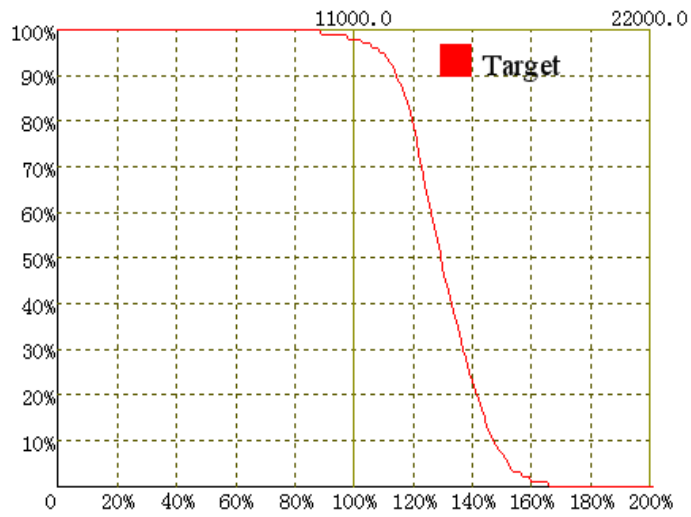

Fig. 5. The target DVH of the treatment plan generated by the DIGH approach for patient 1.

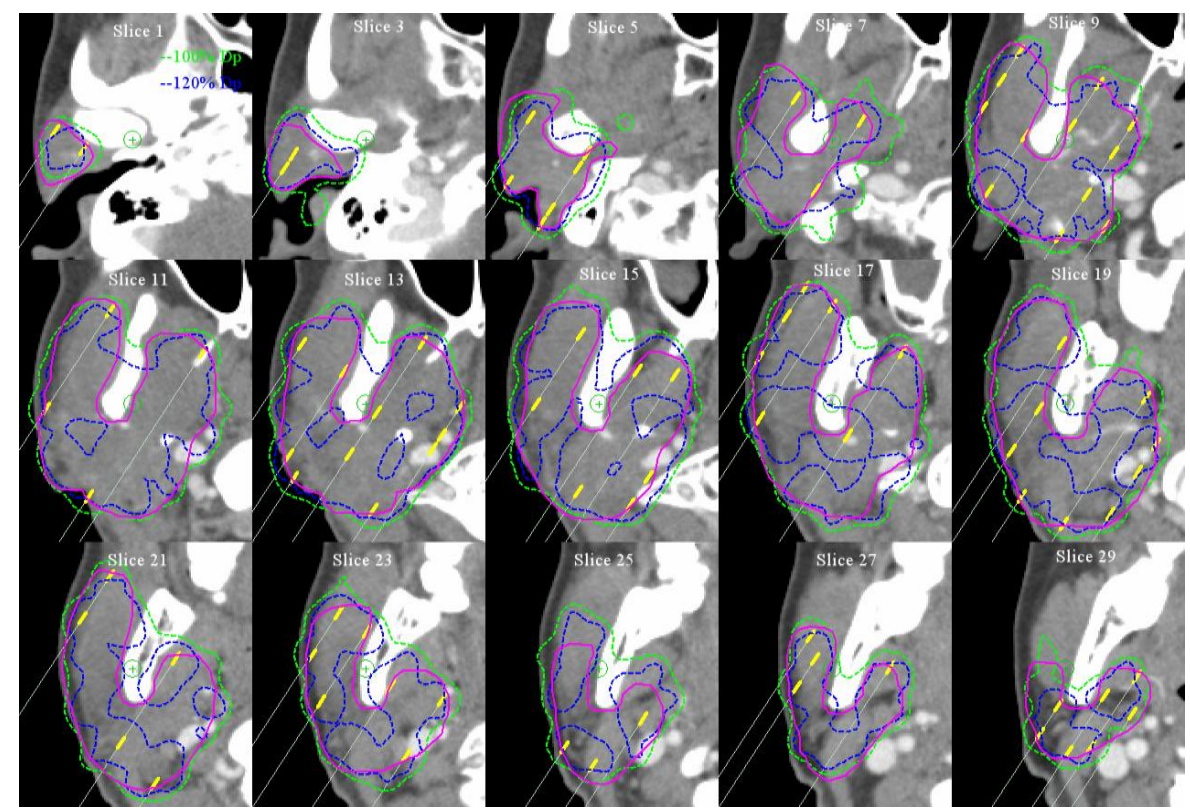

Fig. 6. Seed and dosimetric distribution of the treatment plan generated by the DIGH approach for patient 1. Target contour is outlined in magenta solid line. The isodose lines of $100 \%$ and $120 \%$ Dp are depicted in green and blue, respectively. The selected seeds are marked by solid dots.

As discussed before, OARs are not taken into account during treatment planning. The dosimetric distribution parameters along with computational time, seed and needle numbers are listed in Table 4. Compared with prostate plan, the dose delivered to target volume is more homogeneous. Target volume receiving higher dose is much smaller because of the absence of OARs. As shown in Fig. 5, the DVH curve of target volume drops more quickly. Because target volume is larger and more irregular, adenoid treatment plan uses more seeds and puncture needles, and the computational time is also longer.

The space of puncture holes on template is $5 \mathrm{~mm}$, and the interval between image slices is $2.5 \mathrm{~mm}$. Thus the seeds are distributed on every other slice, see Fig. 6 . As the V150 is rather small, the isodose line of $150 \%$ Dp is not shown.

Despite of the irregularity of target volume, the treatment plan obtained by DIGH approach achieves satisfactory dosimetric distribution, and the DI strategy is also effective on decreasing puncture needles.

\subsection{Mediastinum Treatment Plan}

The issue of whether it is suitable to treat mediastinum tumor by brachytherapy will not be discussed in this paper. As the target volume of mediastinum tumor is extremely large, we use this typical patient case to 
test the robustness of the DIGH approach.

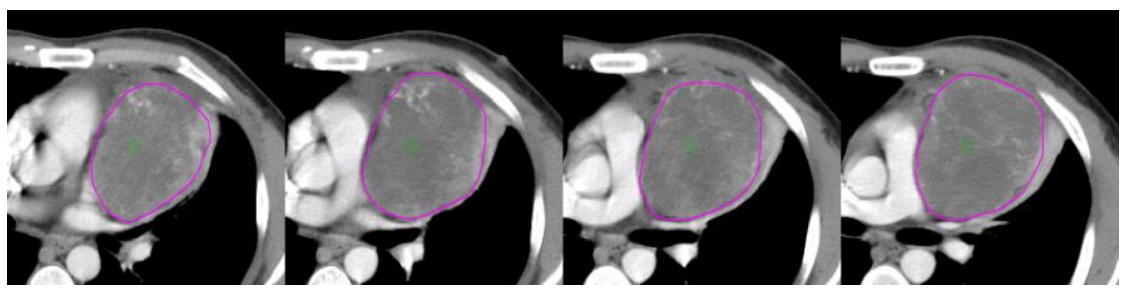

Fig. 7. Adjacent four CT slices of the mediastinum tumor.

Fig. 7 shows the adjacent four slices, in which target volume is outlined by magenta solid line. Because of rib structure, the blocked direction is wide, and the remaining potential seeds after eliminating sheltered seeds are rather few, which are not able to deliver sufficient dose to target volume. And it is inevitable to puncture these blocking ribs when necessary. Therefore the issue of rib blocking is not considered during planning.

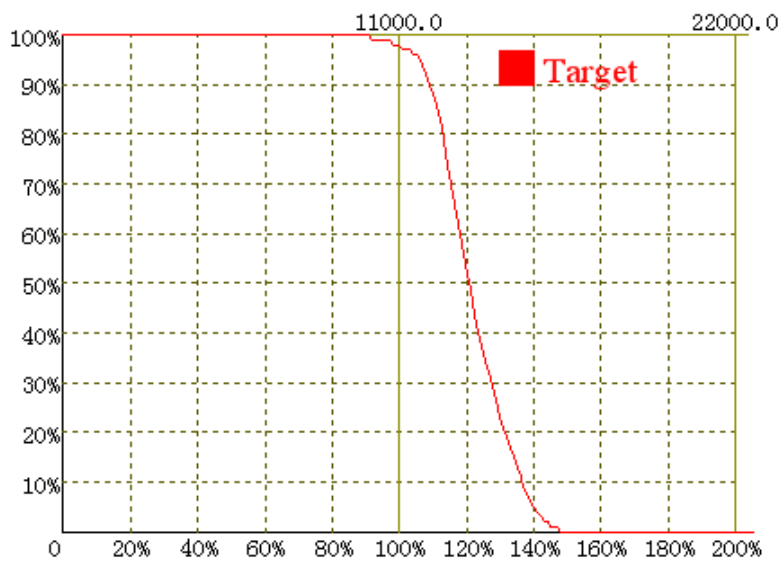

Fig. 8. The target DVH of the treatment plan generated by the DIGH approach for patient 1.

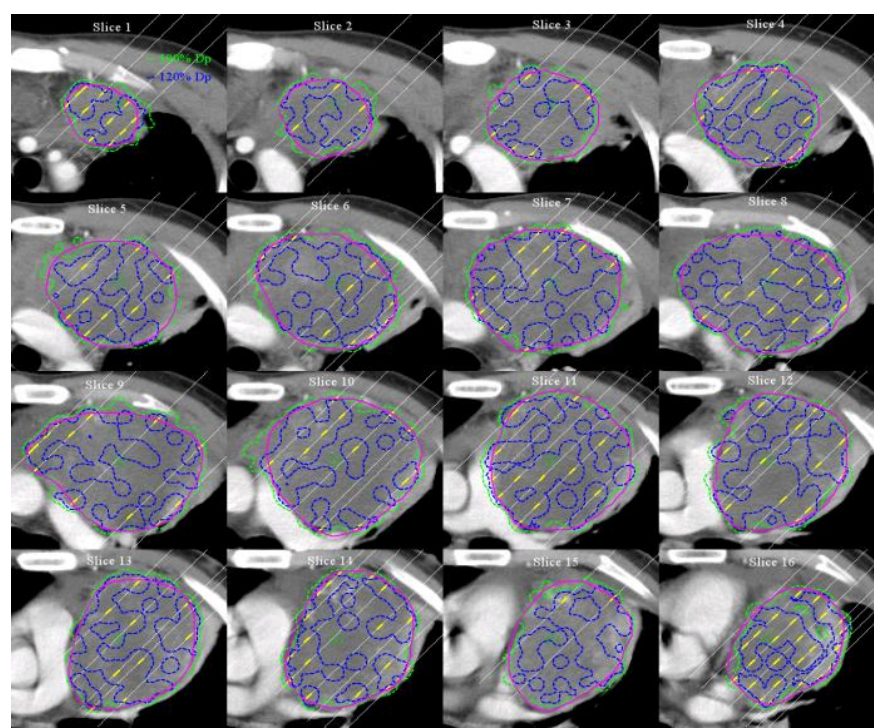

Fig. 9. Seed and dosimetric distribution of the treatment plan generated by the DIGH approach for patient 1. Target contour is outlined in magenta solid line. The isodose lines of $100 \%$ and $120 \%$ Dp are depicted in green and blue, respectively. The selected seeds are marked by solid dots. 
Table 5. Critical Parameters of Mediastinum Treatment Plan

\begin{tabular}{c|c|c|c|c|c|c|c|c|c}
\hline Patient & Vol. & V120 & V100 & DNR & D100 & D90 & Seed & Needle & Time \\
\hline 1 & 337.0 & 52.5 & 97.8 & 0.0026 & 73.7 & 119.9 & 182 & 84 & 26.1 \\
\hline 2 & 316.5 & 45.6 & 98.6 & 0.0018 & 73.7 & 108.2 & 171 & 77 & 24.4 \\
\hline 3 & 322.6 & 47.5 & 99.4 & 0.0032 & 73.7 & 115.4 & 174 & 80 & 25.2 \\
\hline 4 & 306.2 & 56.3 & 98.3 & 0.0034 & 73.7 & 120.3 & 168 & 76 & 23.9 \\
\hline 5 & 345.3 & 56.8 & 98.4 & 0.0041 & 73.7 & 117.5 & 185 & 85 & 28.2 \\
\hline
\end{tabular}

As we discussed before, OARs is also not considered. The DIGH starts after the direction of template is determined. Dosimetric distribution parameters, needle and seed numbers of treatment plan are listed in Table 5. DVH of target volume is shown in Fig. 8. Dosimetric and seed distribution is demonstrated in Fig. 9.

The data and figures indicate that the treatment plan achieves satisfactory conformity and homogeneity, which caters to clinical demands. Considering the large volume of target, seed and needle numbers are also acceptable.

\section{Conclusion and Discussion}

All these treatment plans generated by the DIGH plan achieve satisfactory dosimetric distribution. And the seeds and puncture needles are kept within acceptable level. Computational time of the DIGH approach is proportional to target volume. For the largest one, the computational time is no longer than 30s. Therefore the DIGH is potential for intraoperative planning. Since the DIGH approach is effective for the patient cases of closely adjacent OARs, irregular or extreme large volume target, it is reasonable to assume the approach is potential for more generalized treatment planning of other parts.

To sum up, the most creative part of the DIGH approach is that the clinical considerations about the dosimetric distribution of treatment plans are reasonably "translated" into the seed evaluation criterion. The criterion takes advantage of the prior knowledge about the relative position between the potential seed and critical organs, which makes it possible to generate a feasible treatment plan quickly.

The double iteration strategy is another innovation of the DIGH approach. Based on the feature of heuristic approach, the DI strategy effectively decreases puncture needles without sacrificing the quality of treatment plan.

Due to the issue of bone blocking, the direction of template needs to be adjusted and unfeasible seeds needs to be eliminated before applying the DIGH approach. Our future work is to detect these bones and then determine the optimal template direction and remove the unfeasible seeds based on optimization goals. Also more patient cases will be investigated to further test the DIGH approach in our subsequent work. The needle number of treatment plan is another our concern. Although the needle number is acceptable, we consider it possible to further reduce puncture needles to improve feasibility.

\section{Reference}

[1] Davis, B. J., Horwitz, E. M., et al. (2012). American brachytherapy Society consensus guidelines for transrectal ultrasound-guided permanent prostate brachytherapy. Brachytherapy, 11, 6-19.

[2] Polo, A., Salembier, C., et al. (2010). Review of intraoperative imaging and planning techniques in permanent seed prostate brachytherapy. Radiotherapy and Oncology, 94(1), 12-23. 
[3] Nath, R., Bice, William, S., et al. (2009). AAPM recommendations on dose prescription and reporting methods for permanent interstitial brachytherapy for prostate cancer: Report of Task Group 137. Medical Physics, 36, 5310.

[4] Koukourakis, G., Kelekis, N., et al. (2009). Brachytherapy for prostate cancer: A systematic review. Advances in urology, 2009, 1-12.

[5] Pignol, J.-P., Rakovitch, E., et al. (2009). Tolerance and acceptance results of a palladium-103 permanent breast seed implant Phase I/II study. International Journal of Radiation Oncology Biology Physics, 73(5), 1482-1488.

[6] Keller, B., Sankreacha, R., et al. (2005). A permanent breast seed implant as partial breast radiation therapy for early-stage patients: a comparison of palladium-103 and iodine-125 isotopes based on radiation safety considerations. International Journal of Radiation Oncology Biology Physics, 62(2), 358-365.

[7] Pfister, D. G., Johnson, D. H., et al. (2004). American society of clinical oncology treatment of unresectable non-small-cell lung cancer guideline: Update 2003. Journal of Clinical Oncology, 22(2), 330-353.

[8] Raben, A., \& Mychalczak, B. (1997). Brachytherapy for non-small cell lung cancer and selected neoplasms of the chest. CHEST Journal, 112(4_Supplement), 276S-286S.

[9] Zheng, L., Zhang, J., et al. (2012). Preliminary results of 125I interstitial brachytherapy for locally recurrent parotid gland cancer in previously irradiated patients. Head \& Neck, 34(10), 1445-1449.

[10] Huang, M.-W., et al. (2012). A digital model individual template and CT-guided ${ }^{125}$ I seed implants for malignant tumors of the head and neck. Journal of Radiation Research, 53(6), 973-977.

[11] Zhang, J., et al. (2008). ${ }^{125}$ I seed implant brachytherapy-assisted surgery with preservation of the facial nerve for treatment of malignant parotid gland tumors. International Journal of Oral and Maxillofacial Surgery, 37(6), 515-520.

[12] Pouliot, J., Reinstein, L., et al. (1996). Optimization of permanent 125I prostate implants using fast simulated annealing. International Journal of Radiation Oncology Biology Physics, 36(3), 711-720.

[13] Yu, Y., \& Schell, M. (1996). A genetic algorithm for the optimization of prostate implants. Medical Physics, 23, 2085.

[14] Yu, Y., Zhang, J., et al. (2000). Multi-objective optimization in radiotherapy: applications to stereotactic radiosurgery and prostate brachytherapy. Artificial Intelligence in Medicine, 19(1), 39-51.

[15] Yang, G., Reinstein, L., et al. (1998). A new genetic algorithm technique in optimization of permanent I prostate implants. Medical Physics, 25, 2308.

[16] Lee, E. K., Gallagher, R. J., et al., (1999). Treatment planning for brachytherapy: an integer programming model, two computational approaches and experiments with permanent prostate implant planning. Physics in Medicine and Biology, 44(1), 145.

[17] Lee, E. K., \& Zaider, M., (2003). Mixed integer programming approaches to treatment planning for brachytherapy-application to permanent prostate implants. Annals of Operations Research, 119(1-4), 147-163.

[18] D'Souza, W. D., Meyer, et al. (2001). An iterative sequential mixed-integer approach to automated prostate brachytherapy treatment plan optimization. Physics in Medicine and Biology, 46(2), 297.

[19] Yoo, S., Kowalok, Michael, E., et al. (2003). Treatment planning for prostate brachytherapy using region of interest adjoint functions and a greedy heuristic. Physics in Medicine and Biology, 48(24), 4077.

[20] Yoo, S., Kowalok, M. E., et al. (2007). A greedy heuristic using adjoint functions for the optimization of seed and needle configurations in prostate seed implant. Physics in Medicine and Biology, 52(3), 815.

[21] Chaswal, V., Yoo, S., et al. (2007). Multi-species prostate implant treatment plans incorporating Ir and I 
using a Greedy Heuristic based 3D optimization algorithm. Medical Physics, 34, 436.

[22] Chaswal, V., Thomadsen, B., et al. (2012). Development of an adjoint sensitivity field-based treatment-planning technique for the use of newly designed directional LDR sources in brachytherapy. Physics in Medicine and Biology, 57(4), 963.

[23] Liang, B., Liu, B., et al. (2013). A novel approach to intra-operative planning for prostate brachytherapy. Proceeding of the World Congress on Medical Physics and Biomedical Engineering (pp. 1853-1856), 2012.

[24] Nath, R., Anderson, L., et al. (1995). Dosimetry of interstitial brachytherapy sources: Recommendations of the AAPM radiation therapy committee Task Group No. 43. Medical Physics, 22, 209.

[25] Williamson, J. F., et al. (1999). Guidance to users of Nycomed Amersham and North American Scientific, Inc., I-125 interstitial sources: Dosimetry and calibration changes: recommendations of the American Association of Physicists in medicine radiation therapy committee ad hoc subcommittee on low-energy seed dosimetry. Medical Physics, 26, 570.

[26] Saw, C. B., Suntharalingam, N., et al. (1993). Concept of dose nonuniformity in interstitial brachytherapy. International Journal of Radiation Oncology Biology Physics, 26(3), 519-527.

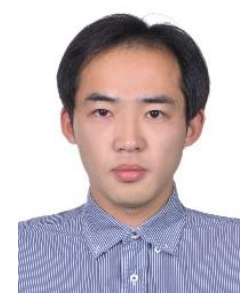

Bin Liang received his bachelor's degree in automation from Hunan University, Changsha, China in 2010. Now he is studying for the doctoral degree of pattern recognition and intelligent system at image processing center of Beihang University, Beijing, China. His doctoral work is on treatment dose calculation and planning optimization for radiotherapy.

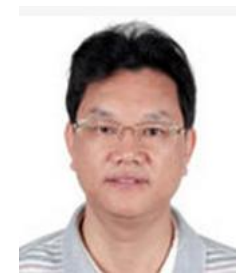

Fugen Zhou received hid B. Eng in electronic engineering from Beihang University, Beijing, China in 1986. He received his M.Eng and Ph.D in pattern recognition and intelligent system at Beihang University in 1989 and 2006. He is the director of image processing center of Beihang University. His research topics include target detection and tracking and recognition, image registration and fusion, medical image processing and recognition, etc.

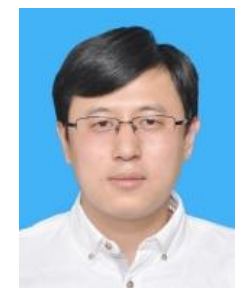

Bo Liu received his Ph.D in pattern recognition and intelligent system at Beihang University, Beijing, China in 2014. His research topics include medical imaging, medical image processing and recognition, etc.

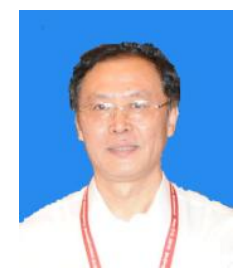

Jianguo Zhang received his M.D in stomatology from Peking University Health Science Center, Beijing, China. He is the vice chairman of the Department of Oral and Maxillofacial Surgery, Peking University, School of Stomatology. 


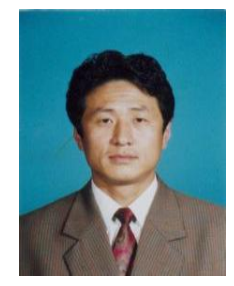

Junjie Wang received his M.D in oncology from Peking University, Beijing, China. He is the director of the Department of Radiation Oncology, CANCER center, Peking University Third Hospital, Beijing, China. His research topics include brachytherapy and IGRT etc.

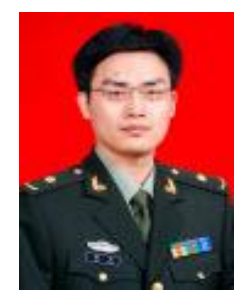

Yong Xu was born in 1971. He is the associate chief physician is the Department of Urology, General Hospital of PLA, Beijing, China. His research topics mainly include the diagnose of urology disease and prostate brachytherapy etc. 